\title{
Effect of RNA Interference-Mediated Suppression of p75 on the Viability of Rat Notochordal Cells
}

\author{
Jong-Beom Park ${ }^{1}$, Dong-Gune Chang ${ }^{2}$, Seung Yeol Oh${ }^{2}$, Eun-Young Park ${ }^{1}$ \\ ${ }^{1}$ Department of Orthopaedic Surgery, Uijeongbu St. Mary's Hospital, College of Medicine, The Catholic University of Korea, Uijeongbu, Korea \\ ${ }^{2}$ Department of Orthopaedic Surgery, Sanggye Paik Hospital, Inje University College of Medicine, Seoul, Korea
}

Study Design: In vitro cell culture model.

Purpose: To investigate the effects of RNA interference (RNAi) on p75 expression and viability of rat notochordal cells treated with serum deprivation.

Overview of Literature: RNAi enables the inhibition of specific genes by sequence-specific gene silencing using a double-stranded RNA.

Methods: Notochordal cells were isolated, cultured, and placed in 10\% (control) or $0 \%$ (apoptosis-promoting) fetal bovine serum (FBS) for 48 hours. The expression of $p 75$, apoptosis, and cell proliferation were determined. To suppress $p 75$ expression, a small interfering RNA (siRNA) was synthesized against p75 (p75 siRNA) and transfected into cells. The suppression of p75 mRNA expression was investigated using the reverse transcription-polymerase chain reaction. The degree of $p 75$ suppression was semiquantitatively analyzed using densitometry. The effect of p75 siRNA on apoptosis and proliferation of cells was determined. Solutions of an unrelated siRNA and transfection agent alone served as controls.

Results: Serum deprivation significantly increased apoptosis by $40.3 \%$, decreased proliferation of notochordal cells by $45.3 \%$ (both, $p<0.001$ ), and upregulated $p 75$ expression. The p75 siRNA suppressed $p 75$ expression in cells cultured in $0 \%$ FBS. The rate of suppression by $p 75$ siRNA of $p 75$ mRNA was $72.9 \%$ ( $p<0.001)$. Suppression of $p 75$ expression by $p 75$ siRNA inhibited apoptosis by $7 \%$ and increased proliferation by $14 \%$ in cells cultured in $0 \%$ FBS (both, $p<0.05)$.

Conclusions: siRNA-mediated suppression of p75 inhibited apoptosis and increased proliferation of notochordal cells under conditions of serum deprivation, suggesting that RNAi might serve as a novel therapeutic approach for disc degeneration caused by insufficient viability of disc cells through the suppression of the expression of harmful genes.

Keywords: RNA interference; p75; Viability; Notochordal cells

\section{Introduction}

Nerve growth factor (NGF) is a member of the neurotrophin family. The biological effects of NGF on cells are mediated by its receptors tropomyosin-related kinase
A (TrkA) and tumor necrosis factor (TNF) family member p75 [1-3]. Similar to other members of the TNF receptor family, the 75 receptor has an intracellular death domain. Therefore, the binding of NGF to the p75 receptor triggers apoptosis in the absence of the TrkA

\footnotetext{
Received Aug 4, 2016; Revised Aug 11, 2016; Accepted Aug 13, 2016

Corresponding author: Jong-Beom Park

Department of Orthopaedic Surgery, Uijeongbu St. Mary's Hospital, College of Medicine,

The Catholic University of Korea, 271 Cheonbo-ro, Uijeongbu 11765, Korea

Tel: +82-31-820-3578, Fax: +82-3-1847-3671, E-mail: spinepjb@catholic.ac.kr
} 
receptor. However, NGF promotes cell survival through the TrkA receptor. The paradoxical and antagonistic responses to NGF are almost completely dependent on the relative abundance of these two distinct NGF receptors [4]. The precise ratio of TrkA and p75 receptors is an important determinant of cell survival and death. The rate of apoptosis in notochordal cells is higher because of caspase activation under conditions of serum deprivation [5]. Further, expression of NGF, p75 receptor, and JNK downstream pathways are upregulated in notochordal cells undergoing apoptosis caused by serum deprivation [6]. Therefore, specific downregulation of p75 might represent a novel therapeutic strategy against disc degeneration caused by insufficient viability of notochordal cells.

RNA interference (RNAi) causes sequence-specific gene silencing through double-stranded RNAs (dsRNAs) $[7,8]$. RNAi involves post-transcriptional gene silencing via a process in which dsRNAs inhibit gene expression through degradation of a specific mRNA. Small interfering RNAs (siRNAs), a component of RNAi, comprise a sense strand as well as an antisense strand that is complementary to a sequence of the suppressed gene [9]. Therefore, synthetic siRNA can trigger an RNAi response in mammalian cells and induce inhibition of specific gene expression. The specificity and potency of synthetic siRNA facilitates elucidation of gene function and investigations of novel approaches to the treatment of disease [10]. Little information is available regarding the application of siRNA technology to the down-regulation of specific genes related to the viability of disc cells.

In the current study, we therefore investigated the effects of siRNA on p75 expression, apoptosis, and proliferation of rat notochordal cells cultured in the absence of serum. An siRNA targeting p75 was synthesized and transfected into notochordal cells for 48 hours under conditions of serum-deprivation, and the effect of siRNA-mediated suppression of p75 on apoptosis and proliferation was investigated.

\section{Materials and Methods}

\section{Notochordal cell culture}

The Animal Care and Use Committee of the author's institution approved all experiments. Lumbar intervertebral discs (L1-L6) were harvested from five male SpragueDawley rats (4 weeks of age) immediately after sacrifice.
We dissected the discs with the aid of a microscope to obtain NP tissues, which were then cultured in Dulbecco's modified Eagle's medium (DMEM, Gibco BRL, Grand Island, NY, USA) containing with $10 \%$ fetal bovine serum (FBS, Hyclone, Ottawa, ON, Canada), $100 \mathrm{U} / \mathrm{mL}$ penicillin (Gibco BRL), and $100 \mathrm{mg} / \mathrm{mL}$ streptomycin (Gibco $\mathrm{BRL}$ ) at $37^{\circ} \mathrm{C}$ for 12 hours in a humidified atmosphere containing $5 \% \mathrm{CO}_{2}$. To isolate notochordal cells, NP tissues in DMEM medium were digested using $0.2 \%$ pronase (Sigma-Aldrich, St. Louis, MO, USA) for 4 hours. After enzymatic digestion, the suspension was filtered through a $70-\mu \mathrm{m}$ mesh (Falcon, Franklin Lakes, NJ, USA). Filtered cells were then washed with DMEM and used as the primary culture. After seven passages, the cells were trypsinized, subcultured into six-well plates $\left(1 \times 10^{6}\right.$ cells per well), and placed in $10 \%$ (control) or $0 \%$ (apoptosis-promoting) FBS for 48 hours.

\section{Terminal deoxynucleotidyl transferase-mediated dUTP nick-end labeling (TUNEL)}

The apoptosis of notochordal cells was determined by incubating them with $15 \mu \mathrm{L}$ of APOPercentage dye (Biocolor Life Science, Carrickfergus, UK) for 30 minutes. After using a syringe to remove the culture medium and dye mixture and then gently washing the cells twice with phosphate buffer saline (PBS; $500 \mu \mathrm{L}$ per well), images of the cells were acquired using an inverted microscope. Notochordal cells cultured for 48 hours were used as the control.

\section{Flow cytometry}

Apoptosis of notochordal cells was determined by treating them with Annexin V-FITC and propidium iodide (PI; PharMingen, San Diego, CA, USA) according to the manufacturer's instructions. Briefly, the cells were washed with cold PBS and then suspended in binding buffer $(10 \mathrm{mM}$ HEPES/NaOH, pH 7.4, $140 \mathrm{mM} \mathrm{NaCl}, 2.5 \mathrm{mM} \mathrm{CaCl}_{2}$ ) $\left(1 \times 10^{6}\right.$ cells per well). Cells were treated with $5 \mu \mathrm{L}$ each of Annexin V-FITC and PI and then analyzed 48 hours later using a FACScan flow cytometer (Becton Dickinson, San Jose, CA, USA).

\section{Cell proliferation assay}

The proliferation of notochordal cells was determined 
using 3-(4,5-dimethylthiazol-2-yl)-5-(3-carboxymethoxy-phenyl)-2-(4-sulfophenyl)-2H-tetrazolium, inner salt (MTS) (CellTiter 96 AQueous One Solution Cell Proliferation assay; Promega, Madison, WI, USA). Notochordal cells treated with media supplemented with $10 \%$ FBS or in media without FBS (0\% FBS) were added to the wells of a 96-well plate. The medium was equilibrated for 1 hour and then $20 \mu \mathrm{L}$ of MTS and phenazine methosulfate were added to each well. After incubation for 2 hours at $37^{\circ} \mathrm{C}$ in a humidified atmosphere containing $5 \% \mathrm{CO}_{2}$, absorbance at $490 \mathrm{~nm}$ was recorded using a spectrophotometric plate reader. Each value shown in the figures represents the mean \pm standard deviation of six replicates.

\section{Western blot analysis of p75}

p75 levels in notochordal cells were determined using western blot analysis according to the manufacturer's instructions (Santa Cruz Biotechnology Inc., Paso Robles, CA, USA). Notochordal cells were washed with ice-cold PBS and lysed in protein lysis buffer containing $50 \mathrm{mM}$ HEPES, pH 7.5, $150 \mathrm{mM} \mathrm{NaCl}, 1.5 \mathrm{mM} \mathrm{MgCl}, 1 \mathrm{mM}$ ethylene glycol tetraacetic acid, $10 \%$ glycerol, $1 \%$ Triton $\mathrm{X}-100$, and $1 \mu \mathrm{M}$ phenylmethylsulfonyl fluoride. Cell lysates were centrifuged at $12,000 \times \mathrm{g}$ for 15 minutes, and protein concentrations were measured using the bicinchoninic acid method (Thermo Fisher Scientific, Pittsburgh, PA, USA). Proteins (50 $\mu$ g per lane) were separated using $10 \%$ sodium dodecyl sulfate-polyacrylamide gel electrophoresis and electrophoretically transferred onto a nitrocellulose membrane. The membranes were incubated with primary antibodies against Fas (Santa Cruz Biotechnology Inc.) and then with a horseradish peroxidaseconjugated IgG secondary antibody (Bio-Rad, Richmond, CA, USA). Immunoreactive bands were visualized using an enhanced chemiluminescence detection kit (Santa Cruz Biotechnology Inc.).

\section{Transfection of notochordal cells with a p75-specific siRNA}

Notochordal cells were cultured to $80 \%-85 \%$ confluence on the day of transfection. The siRNA constructs (Dharmacon, Thermo Fisher Scientific) used in the present study were p75 siRNA (sense, GAACAUAUAGACUCCUUUAUU) and a negative siRNA (sense, UAGC-
GACUAAACACAUCAA). The cells were transfected with siRNA using an siRNA transfection reagent (DharmaFECT; Dharmacon) according to the manufacturer's instructions. Transfections were performed in serum-free alpha minimum essential medium containing 200 nmole of siRNA and $6 \mu \mathrm{L}$ of DharmaFECT (Dharmacon). The GeneBank accession number of the p75 cDNA sequence used to generate the p75 siRNA is NM012610. After 18 hours, the transfection medium was replaced with complete medium, and the cells were collected after 48 hours. The negative siRNA and transfection agent alone (MOCK) were used as controls.

\section{7. siRNA-mediated suppression of p75 mRNA expression}

Total RNA was extracted using with TRIzol regent (Invitrogen, Grand Island, NY, USA) from notochordal cells according to the manufacturer's instructions. For cDNA synthesis, $2 \mu \mathrm{g}$ of total RNA were reverse transcribed in a reaction mixture containing 25 units of ribonuclease inhibitor, 15 units of reverse transcriptase, $500 \mathrm{ng}$ of oligo(dT) primer, $3 \mathrm{mM} \mathrm{MgCl}, 0.5 \mathrm{~mm} \mathrm{dNTP}$, and $1 \times$ RT buffer (Promega). The cDNA was used as a template for polymerase chain reaction (PCR) using GoTaq Polymerase (Promega) and PCR primers that amplify p75 (Bioneer, Cheongwon, Korea). PCR was performed using a Mycycler thermal cycler (Bio-Rad, Hercules, CA, USA) according to the manufacturer's instructions. Analysis of the PCR products using $1 \%$ agarose gel electrophoresis revealed single amplicons of the expected sizes. The primer sequences and PCR conditions are summarized in Tables 1 and 2. Blots were analyzed using an Imaging Densitometer GF670 and Molecular Analyst software (Bio-Rad), and the results are expressed relative to those of glyceraldehyde 3-phosphate dehydrogenase (GAPDH) mRNA. All experiments were repeated three times per sample, and the data represent averaged values.

Table 1. The primer sequences of polymerase chain reaction

\begin{tabular}{llc} 
Primer & \multicolumn{1}{c}{ Sequence } & Size (bp) \\
p75 & 5'-GTGCCTATGGCTACTACCAG-3' & 499 \\
& 5'-AGATGGAGCAATAGACAGGA-3' & \\
GAPDH & 5'-ATCATCTCCGCCCCTTCTGC-3' & 437 \\
& 5'-GCCTGCTTCACCACCTTCTT-3' & \\
\hline
\end{tabular}

GAPDH, glyceraldehyde 3-phosphate dehydrogenase. 
Table 2. The experimental conditions of polymerase chain reaction

\begin{tabular}{|c|c|c|c|c|}
\hline \multirow{2}{*}{ Primer } & \multicolumn{3}{|c|}{ Experimental conditions } & \multirow{2}{*}{ Cycle } \\
\hline & Denaturation & Annealing & Polymerization & \\
\hline p75 & $94^{\circ} \mathrm{C} 5 \mathrm{~min}$ & $94^{\circ} \mathrm{C} 60 \mathrm{sec} \rightarrow 53.7^{\circ} \mathrm{C} 60 \mathrm{sec} \rightarrow 72^{\circ} \mathrm{C} 60 \mathrm{sec}$ & $72^{\circ} \mathrm{C} 5 \min$ & 35 \\
\hline GAPDH & $94^{\circ} \mathrm{C} 5 \min$ & $94^{\circ} \mathrm{C} 30 \mathrm{sec} \rightarrow 56^{\circ} \mathrm{C} 30 \mathrm{sec} \rightarrow 72^{\circ} \mathrm{C} 30 \mathrm{sec}$ & $72^{\circ} \mathrm{C} 5 \min$ & 25 \\
\hline
\end{tabular}

GAPDH, glyceraldehyde 3-phosphate dehydrogenase.

\section{Effect of siRNA-mediated suppression of p75 on apoptosis and proliferation}

TUNEL, flow cytometry, and an MTS assay were used to investigate the effects of siRNA-mediated suppression of p75 expression on apoptosis and proliferation of notochordal cells using the methods described above. Statistical analysis was performed using a $t$ test, and $p<0.05$ was considered statistically significant.

\section{Results}

\section{Increased apoptosis and decreased proliferation of notochordal cells is associated with upregulation of p75 under conditions of serum deprivation}

TUNEL and flow cytometry results are displayed in Fig. 1A. Notochordal cells incubated in 0\% FBS for 48 hours displayed a significantly greater rate of apoptotic death compared with cells incubated in 10\% FBS $(5.9 \% \pm 1.1 \%$ vs. $46.2 \% \pm 3.5 \%, p<0.001$ ) (Fig. 1B). MTS assays showed that notochordal cells incubated in 0\% FBS for 48 hours displayed reduced proliferation ratios compared with those incubated in $10 \%$ FBS $(1.111 \pm 0.24$ vs. $0.608 \pm 0.12$, $p<0.001$ ) (Fig. 2). Western blot analysis demonstrated that p75 expression was upregulated in cells incubated in $0 \%$ FBS compared with those cultured in 10\% FBS (Fig. 3). These results strongly suggest that upregulation of p75 expression induced by serum deprivation was responsible for increased apoptosis and decreased proliferation of notochordal cells.

\section{2. siRNA-mediated suppression of p75 in notochordal cells}

Reverse transcription polymerase chain reaction results are shown in Fig. 4. The p75 siRNA significantly suppressed p75 mRNA levels in notochordal cells cultured in
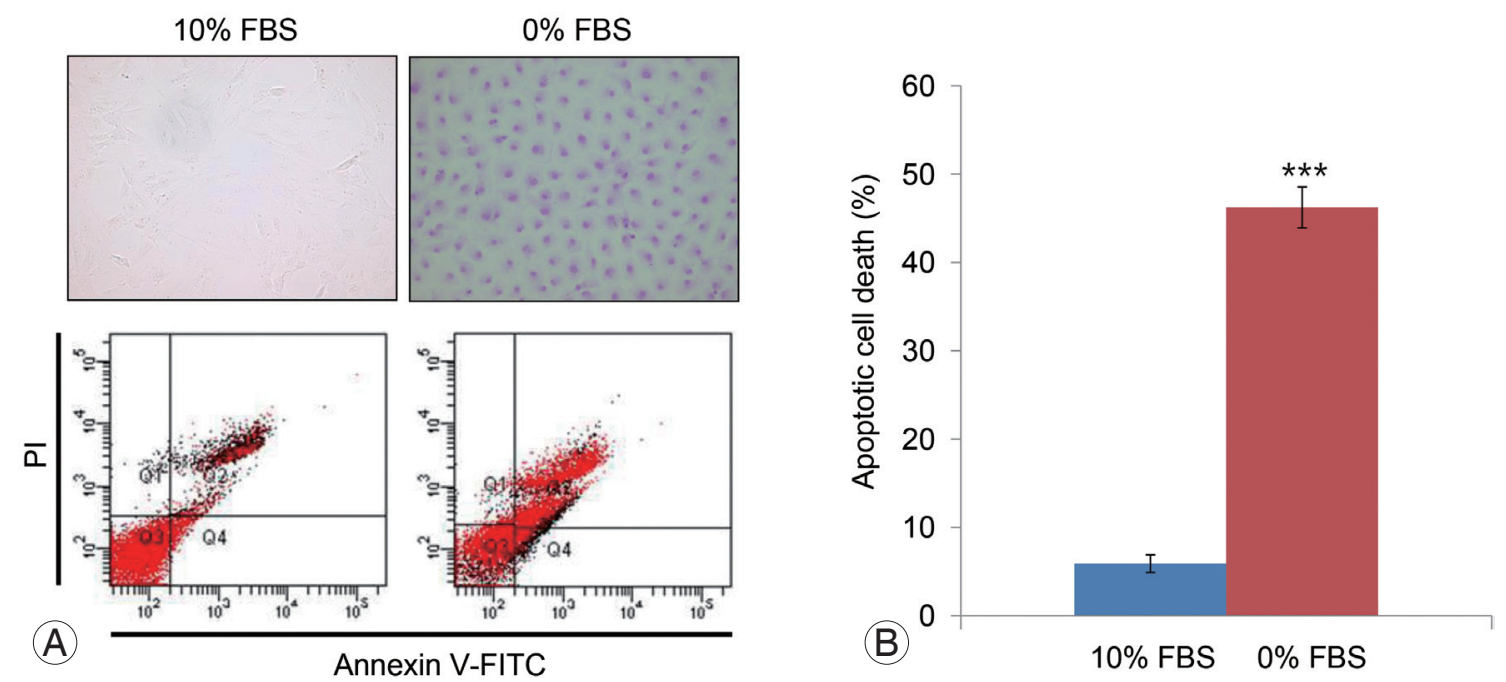

Fig. 1. (A) TUNEL and flow cytometry results are displayed (×400). (B) Notochordal cells incubated in $0 \%$ FBS for 48 hours displayed a significantly greater rate of apoptotic cell death by $40.3 \%$ compared with cells incubated in $10 \%$ FBS $(5.9 \pm 1.1 \%$ vs. $46.2 \% \pm 3.5 \%, p<0.001)$. TUNEL, terminal deoxynucleotidyl transferase-mediated dUTP nick-end labeling; FBS, fetal bovine serum; Pl, propidium iodide. ${ }^{* * *} p<0.001$. 


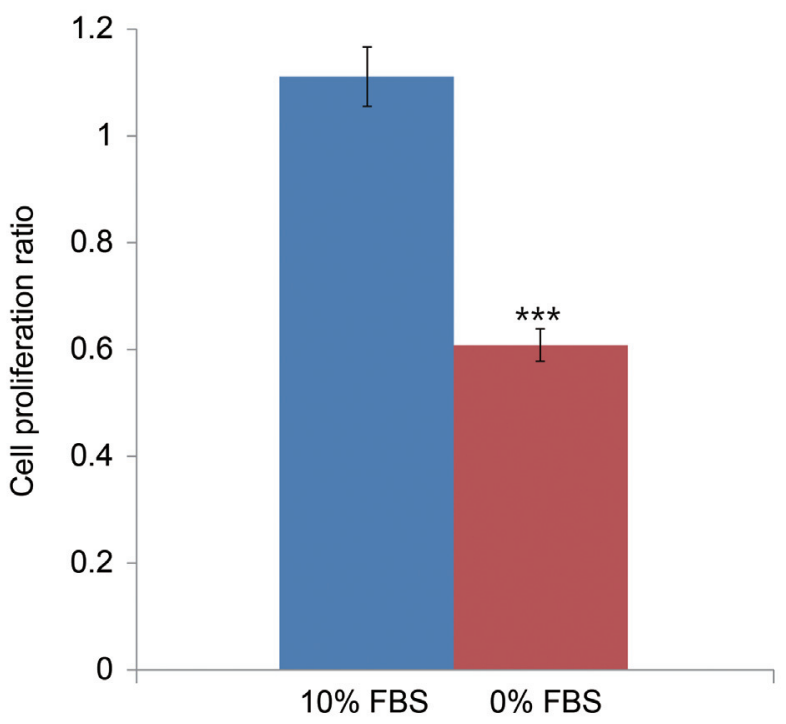

Fig. 2. MTS proliferation assay showing that notochordal cells incubated in 0\% FBS for 48 hours displayed reduced proliferation ratios by $45.3 \%$ compared with those incubated in $10 \%$ FBS $(1.111 \pm 0.24$ vs. $0.608 \pm 0.12, p<0.001)$. MTS, 3-(4,5-dimethylthiazol-2-yl)-5-(3carboxymethoxyphenyl)-2-(4-sulfophenyl)-2H-tetrazolium; FBS, fetal bovine serum. ${ }^{* *} p<0.001$

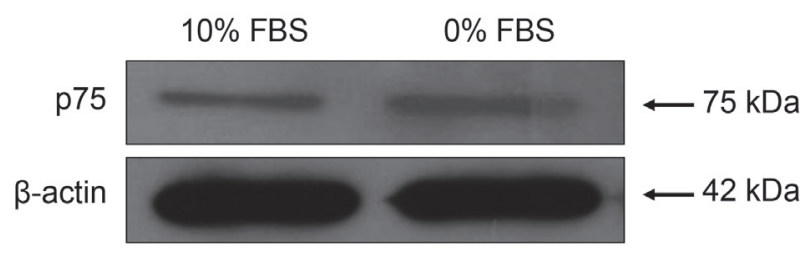

Fig. 3. Western blot analysis demonstrating that p75 expression was upregulated in cells incubated in 0\% FBS compared with those cultured in $10 \%$ FBS. FBS, fetal bovine serum.

$0 \%$ FBS (Fig. 4A). The rate of suppression of p75 mRNA mediated by the p75 siRNA was $72.9 \%(p<0.001)$ (Fig. $4 B)$.

\section{Effect of siRNA-mediated suppression of p75 on apoptosis and proliferation of notochordal cells}

TUNEL demonstrated that p75 siRNA significantly decreased apoptotic cell death of notochordal cells cultured in $0 \%$ FBS (Fig. 5). Flow cytometry results are displayed in Fig. 6. The p75 siRNA significantly inhibited apoptosis by $7 \%$ in cells cultured in $0 \%$ FBS $(46.2 \% \pm 3.5 \%$ vs. $39.2 \% \pm 3.1 \%, p<0.05)$, and p75 siRNA significantly increased the proliferation of cells cultured in $0 \%$ FBS by $14 \%(0.608 \pm 0.12$ vs. $0.693 \pm 0.16, p<0.05)$ (Fig. 7$)$.

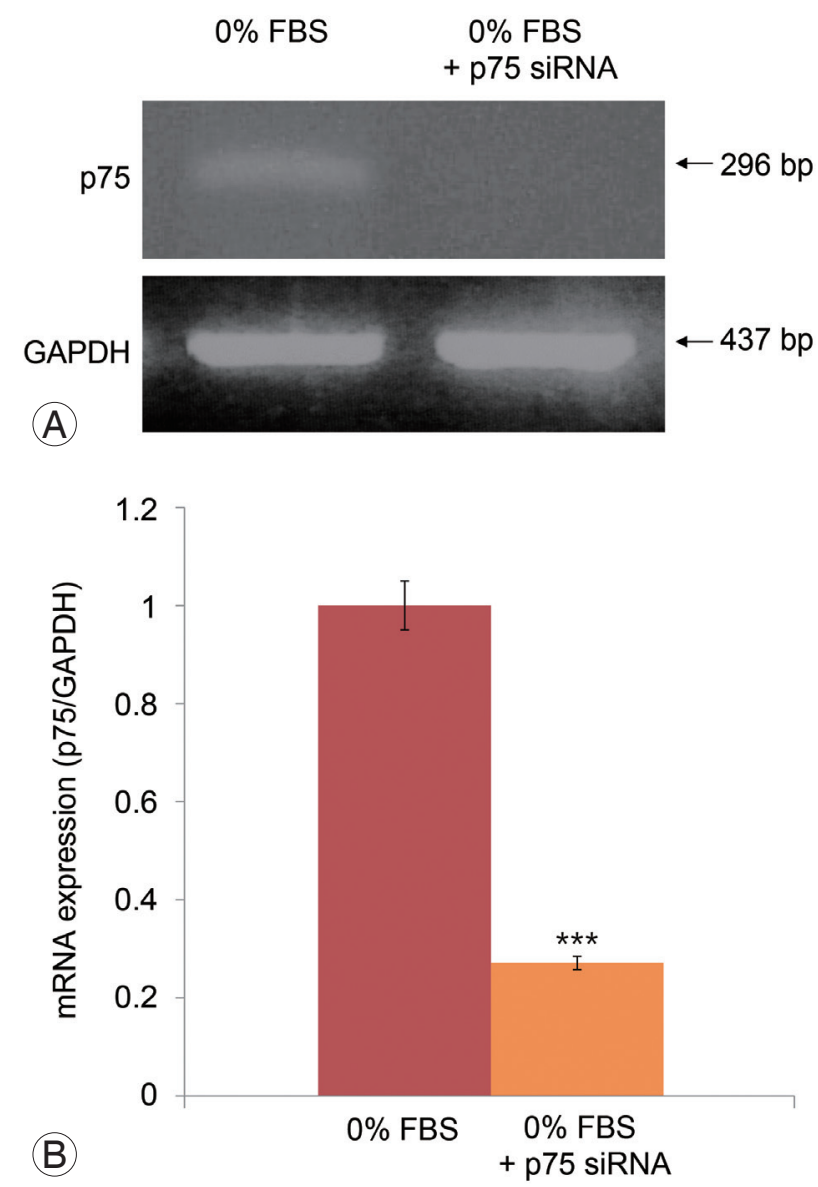

Fig. 4. (A) Reverse transcription-polymerase chain reaction results. (B) p75 siRNA significantly suppressed p75 mRNA levels in notochordal cells cultured in $0 \%$ FBS. The rate of suppression of p75 mRNA by siRNA was $72.9 \%(p<0.001)$. siRNA, small interfering RNA; FBS, fetal bovine serum; GAPDH, glyceraldehyde 3-phosphate dehydrogenase. ${ }^{* * *} p<0.001$.
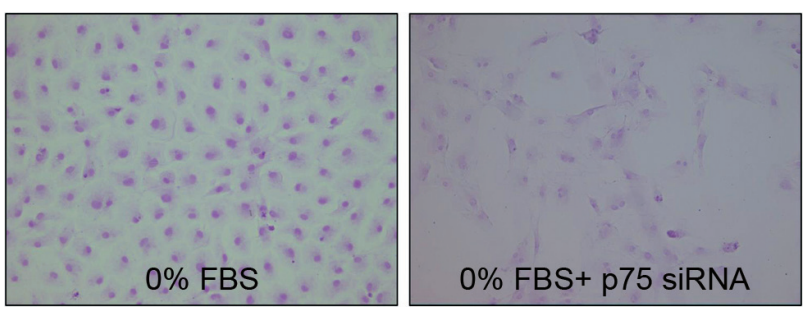

Fig. 5. TUNEL demonstrating that p75 siRNA significantly decreased apoptotic death of notochordal cells cultured in 0\% FBS. TUNEL, terminal deoxynucleotidyl transferase-mediated dUTP nick-end labeling; siRNA, small interfering RNA; FBS, fetal bovine serum. (×400).

\section{Discussion}

The current study demonstrates that serum deprivation increased apoptosis and decreased proliferation of noto- 


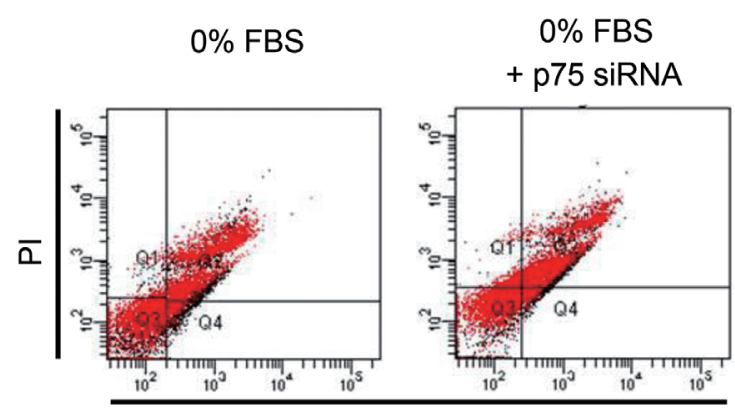

(A)
Annexin V-FITC

Fig. 6. (A) Flow cytometry results. (B) p75 siRNA significantly inhibited apoptosis by $7 \%$ in notochordal cells cultured in $0 \%$ FBS (46.2\% $\pm 3.5 \%$ vs. $36.9 \% \pm 2.7 \%, p<0.05)$. siRNA, small interfering RNA; FBS, fetal bovine serum; PI, propidium iodide. ${ }^{*} p<0.05$.

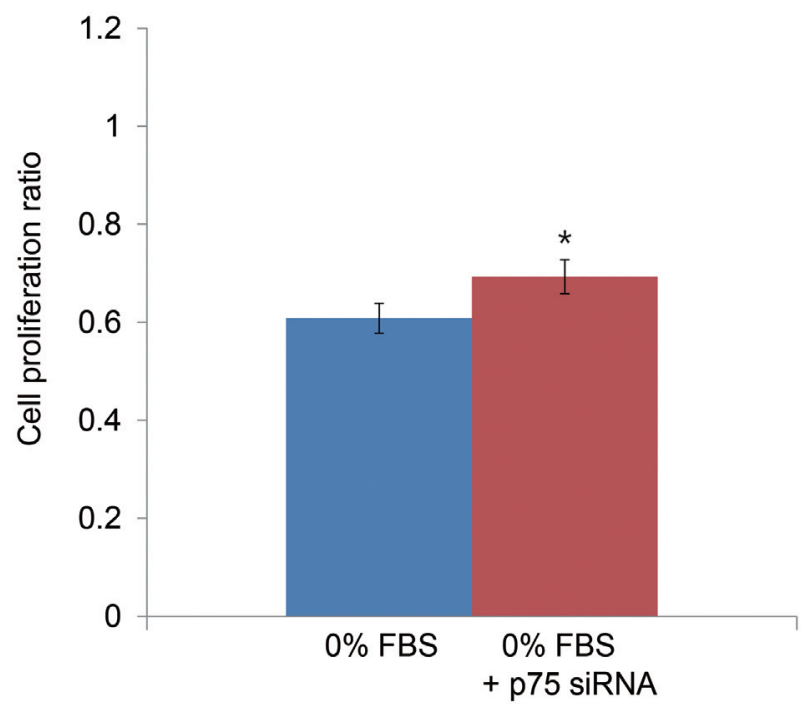

Fig. 7. MTS proliferation assay showing that p75 siRNA increased proliferation by $14 \%$ in notochordal cells cultured in $0 \%$ FBS $(0.608 \pm 0.12$ vs. $0.693 \pm 0.16, p<0.05)$. MTS, 3-(4,5-dimethylthiazol-2-yl)-5-(3carboxymethoxyphenyl)-2-(4-sulfophenyl)-2H-tetrazolium; siRNA, small interfering RNA; FBS, fetal bovine serum. ${ }^{*} p<0.05$.

chordal cells associated with upregulation of $\mathrm{p} 75$. These results strongly suggest that upregulation of p75 expression is responsible for increased apoptosis and decreased proliferation of notochordal cells, which leads to disc degeneration. Therefore, we believe that specific downregulation of p75 by siRNA represents a potential therapeutic approach to disc degeneration due to insufficient viability of notochordal cells. To evaluate this hypothesis, siRNA targeting p75 was synthesized and transfected into notochordal cells using oligonucleotides for 48 hours un- der serum-deprivation conditions. The p75 siRNA inhibited the expression of p75 mRNA in notochordal cells by approximately $72.9 \%$. We believe that this inhibitory effect was more rapid and effective than expected, because total serum deprivation inhibits cell survival. Therefore, our results suggest that siRNA silencing of the p75 gene will facilitate investigations of the apoptotic pathways of disc cells associated with disc degeneration. Selective silencing of endogenous genes by siRNA is utilized extensively in several studies [7-10]. The advantages of this technology are that synthetic siRNAs are relatively easy to produce and apply to cells. Moreover, siRNA is efficient, because a single dose of siRNA can sustain RNAi long enough to allow recovery of cellular regulatory systems $[9,10]$. According to our literature review, the present study is the first to demonstrate RNAi-mediated suppression of p75 expression in disc cells, including notochordal cells.

To repair degenerated discs, several antiapoptotic agents were investigated for their ability to attenuate or prevent apoptosis of disc cells under various experimental conditions $[11,12]$. Growth factors, such as platelet-derived growth factors, insulin-like growth factor-1 (IGF-1), and NGF, exert prosurvival, antiapoptotic effects on disc cells $[13,14]$. However, the range of antiapoptotic effects of growth factors varies from $0.5 \%$ to $5 \%$ depending on the experimental conditions and dosages, although cocktail therapy using growth factors increases the antiapoptotic effect to $9 \%$ [14]. The optimal dosage of each growth factor for reducing apoptosis of disc cells is unclear. Moreover, the specific mechanisms, downstream pathways, or both related to the antiapoptotic effect of each growth fac- 
tor should be investigated.

Apoptosis is mediated by the activation of caspases. Caspases act as either initiators (caspase- 8 or caspase-9) or as a common executioner (caspase-3) of apoptosis. Therefore, an alternative strategy involves interfering with caspase activation. For example, caspase inhibitors attenuate apoptosis of disc cells $[15,16]$. However, caspase inhibitors block apoptosis after its initiation or at a late stage. Therefore, targeting caspase activation may not be effective, because activation of early apoptotic signals may cause detrimental effects on disc cell metabolism and activity. Moreover, caspase activation differs depending on the apoptotic stimulus, even in the same cells. These limitations suggest that siRNA technology can be used to target the early stage of apoptosis by acting before caspase activation.

Therefore, we investigated the effects of siRNA-mediated suppression of p75 on apoptosis and proliferation of notochordal cells under conditions of serum deprivation. siRNA-mediated suppression of p75 expression significantly inhibited apoptosis by approximately $7 \%$. The efficacy of therapeutic inhibition of apoptosis by p75 siRNA in the present study was superior to that of growth factors. For example, the rate of inhibition of apoptosis (7\%) by p75 siRNA was higher compared with that induced by NGF and IGF-1 (2\% and 5\%) [14]. Further, siRNAmediated suppression of p75 expression increased cell proliferation by approximately $14 \%$. These results suggest that down-regulation of p75 expression at the initiation stage of apoptosis attenuated or delayed the onset of apoptosis and increase proliferation, leading to enhanced viability of notochordal cells. Therefore, we believe that these dual positive effects of p75 siRNA might represent a novel therapeutic approach for disc degeneration caused by insufficient viability of disc cells caused by the suppression of the expression of harmful genes.

There are some limitations to the current study. The first is that this study investigated an early phase in vitro, and animal studies must be performed to support our conclusion that we revealed a novel therapeutic approach for disc degeneration. The second limitation is that early and late apoptotic events were not distinguished. Finally, translation to the clinic seems limited because of the smaller beneficial effects on apoptosis inhibition (7\%) and increased proliferation (14\%). However, considering that $0 \%$ serum deprivation is extremely detrimental, we are certain that the therapeutic effects of Fas siRNA will be increased using more favorable situations compared with cell culture media containing 10\% FBS.

\section{Conclusions}

In conclusion, siRNA-mediated suppression of p75 expression inhibited apoptosis and increased the proliferation of notochordal cells under conditions of serum deprivation. These results suggest that RNAi technology represents a novel therapeutic approach for disc degeneration caused by insufficient viability of disc cells, which is associated with the suppression of the expression of harmful genes.

\section{Conflict of Interest}

No potential conflict of interest relevant to this article was reported.

\section{Acknowledgments}

Partial support of 2016 AOSpine Korea research project.

\section{References}

1. Park JB, Lee CK, Koh JS, Lee JK, Park EY, Riew $\mathrm{KD}$. Overexpressions of nerve growth factor and its tropomyosin-related kinase A receptor on chordoma cells. Spine (Phila Pa 1976) 2007;32:1969-73.

2. Rabizadeh S, Oh J, Zhong LT, et al. Induction of apoptosis by the low-affinity NGF receptor. Science 1993;261:345-8.

3. Frade JM, Barde YA. Nerve growth factor: two receptors, multiple functions. Bioessays 1998;20:137-45.

4. Friedman WJ, Greene LA. Neurotrophin signaling via Trks and p75. Exp Cell Res 1999;253:131-42.

5. Suhl KH, Park JB, Park EY, Rhee SK. Effect of nerve growth factor and its transforming tyrosine kinase protein and low-affinity nerve growth factor receptors on apoptosis of notochordal cells. Int Orthop 2012;36:1747-53.

6. Park EY, Park JB. Dose- and time-dependent effect of high glucose concentration on viability of notochordal cells and expression of matrix degrading and fibrotic enzymes. Int Orthop 2013;37:1179-86.

7. Miao GY, Lu QM, Zhang XL. Downregulation of survivin by RNAi inhibits growth of human gastric 
carcinoma cells. World J Gastroenterol 2007;13:11704.

8. Rossi A, Ciafre S, Balsamo M, Pierimarchi P, Santoro MG. Targeting the heat shock factor 1 by RNA interference: a potent tool to enhance hyperthermochemotherapy efficacy in cervical cancer. Cancer Res 2006;66:7678-85.

9. Tuschl T, Borkhardt A. Small interfering RNAs: a revolutionary tool for the analysis of gene function and gene therapy. Mol Interv 2002;2:158-67.

10. Zender L, Kubicka S. SiRNA based strategies for inhibition of apoptotic pathways in vivo: analytical and therapeutic implications. Apoptosis 2004;9:51-4.

11. Zhao CQ, Jiang LS, Dai LY. Programmed cell death in intervertebral disc degeneration. Apoptosis 2006;11: 2079-88.

12. Choi YS. Pathophysiology of degenerative disc disease. Asian Spine J 2009;3:39-44.
13. Gruber HE, Norton HJ, Hanley EN Jr. Anti-apoptotic effects of IGF-1 and PDGF on human intervertebral disc cells in vitro. Spine (Phila Pa 1976) 2000; 25:2153-7.

14. Park JB, Kim YB, Park EY. Synergistic effect of nerve growth factor and insulin-like growth factor-1 on providing a pro-survival, anti-apoptotic benefit and increased extracellular matrix synthesis in stressed rat intervertebral disc cells. J Neurol Sci 2015;32:72837.

15. Kim KW, Ha KY, Lee JS, Rhyu KW, An HS, Woo YK. The apoptotic effects of oxidative stress and antiapoptotic effects of caspase inhibitors on rat notochordal cells. Spine (Phila Pa 1976) 2007;32:2443-8.

16. Park JB, Park IC, Park SJ, Jin HO, Lee JK, Riew KD. Anti-apoptotic effects of caspase inhibitors on rat intervertebral disc cells. J Bone Joint Surg Am 2006;88: 771-9. 